Asian Clinical Symposium

Regional Differences in Atherosclerotic Risk Factors and Cardiovascular Outcomes in Asian Population

\title{
Risk Factors of Cardiovascular Disease in China
}

Chongyou Lee

Cardiology, Peking University People’s Hospital, Beijing, China

J Atheroscler Thromb, 2014; 21:S29.

This topic was presented at the Asian Clinical Symposium which was held during the 44th Annual Scientific Meeting of the Japan Atherosclerosis Society on July 20, 2012 in Fukuoka, Japan. 